\title{
Wandlungen der japanischen Verkehrsstruktur
}

Wenige Jahre nach der Meiji-Restauration wurde am 14. Oktober 1872 die erste japanische Eisenbahnlinie zwischen der Station Shimbashi (Tokyo) und Yokohama eröffnet. Rasch überzog ein die Transportbedürfnisse befriedigendes Liniennetz alle Inseln, die mit Fährediensten verbunden wurden. Anfänglich waren die meisten Bahnen Privatunternehmen; nach der Jahrhundertwende (31.3.1906) wurden die wichtigsten Gesellschaften nationalisiert und seit 1.6. 1949 als eine "public corporation" (JNR = Japanese National Railways) betrieben. Eisenbahnen besassen im Landverkehr lange praktisch ein Transportmonopol; damit sie dieses nicht missbrauchen würden, können Aenderungen an den Personen- und Frachttarifen nur durch das Parlament beschlossen werden. Noch 1955 bewältigten die JNR (in t-km gemessen) $50 \%$ des Güterverkehrs. Die privaten Gesellschaften meist Vorortsbahnen - hatten nur 1\% und die Strasse 11\% Anteil. Ebenfalls bedeutend war mit $38 \%$ die Küstenschiffahrt.

Die japanischen Eisenbahnen wurden als Schmalspurbahnen (Kapspur, $1067 \mathrm{~mm}$ ) gebaut, was sich auf ihre Leistungsfähigkeit ungünstig auswirkt. 1975 lagen beispielsweise die fahrplanmässigen Geschwindigkeiten je nach Zugskategorie im Passagierverkehr zwischen 35 und $86 \mathrm{~km} / \mathrm{h}$, beim Güterverkehr zwischen 27 und $52 \mathrm{~km} / \mathrm{h}$. Als in der Nachkriegszeit der wirtschaftliche Aufschwung verbunden mit einer Konzentration der Bevölkerung und aller wirtschaftlichen Aktivitäten im Raume der japanischen Megalopolis (Tokyo-Nagoya-Osaka/ Kobe-Kita Kyushu) einsetzte, genügten die bestehenden Bahnen den Anforderungen nicht mehr und die gesamte bisher recht einfache Verkehrsstruktur wandelte sich in zehn bis fünfzehn Jahren von Grund auf.

Der Vorortsverkehr in den rasch wachsenden Grossstädten nahm sprunghaft zu. Als Beispiel diene Tokyo: Von 1965 bis 1974 stieg er bei den JNR auf $116 \%$, bei den privaten Gesellschaften auf $131 \%$ und bei der Untergrundbahn auf $225 \%$. Die vorgeschriebenen Fahrgastkapazitäten werden im Spitzenverkehr in unerträglicher Weise überschritten: 1974 wiesen wichtige Linien mittlere Auslastungen von 259,269 und $289 \%$ auf! Von $250 \%$ an ist man festgekeilt und von $300 \%$ an wird Atemnot festgestellt und Körperverletzungen ( $z$. B. durch Fensterbruch) sind nicht selten. Pendlerdistanzen betragen oft eine, manchmal zwei Stunden. Die im Vorortsverkehr wichtigen Privatgesellschaften hatten 1974 am gesamten Personenverkehr noch $16 \%$ Anteil, während ihr Güterverkehr auf Null absank. Auch auf den wichtigen interurbanen Hauptlinien (meist JNR) wurden die Züge immer mehr überfüllt. Nur auf unrentablen Nebenbahnen konnte man ohne Reservation noch auf einen Sitzplatz hoffen. Auf solchen Lokallinien nahm der Personenverkehr von 1965 - 1974 sogar ab, während er auf den Expresslinien auf $161 \%$ zunahm. Hier hatte die Streckenbelastung ihr Maximum erreicht.

1959 wurde mit dem Bau einer neuen Stammlinie (Shin-kan-sen) von Tokyo vorerst bis Osaka begonnen (eröffnet 1.10.1964), die am 15. 5. 1972 bis Okayama und am 10.3.1975 bis Hakata (Kyushu) weitergeführt wurde. Im Aus land wurde vor allem bekannt, dass auf dieser Linie Geschwindigkeiten bis zu $256 \mathrm{~km} / \mathrm{h}$ erreicht wurden (fahrplanmässig $\max .210 \mathrm{~km} / \mathrm{h}$ ). Dadurch rückten sich diese Orte näher, was in Werbeschriften durch entsprechende "mental maps" der in ihren Dimensionen veränderten japanischen Inselwelt zum Ausdmuck gebracht wird (Fig. 1). Im Rahmen unserer Betrachtung ist vielleicht wichtiger, dass diese Normalspurlinie ausschliesslich dem Personenverkehr dient und sich die Züge - jeder einzelne besitzt eine Kapazität von 1500 Passagieren - mit einer Frequenz von je zehn in der Stunde folgen. In gleicher Weise wie in einer Mittelstadt die Strassenbahn erfüllt Shinkan-sen innerhalb der Megalopolis eine spezielle Verkehrsfunktion. Sie ist mit mund $20 \%$ Anteil am gesamten Personenverkehr auch dafür verantwortlich, dass im Passagierver-. kehr die JNR 1974 immer noch fast einen Drittel des Verkehrs bewältigten. Im Güter-

BOESCH Hans, Prof. Dr. , Geographisches Institut Universität Zürich, Blümlisalpstrasse 10, 8006 Zürich 
verkehr veränderte sich die Transportleistung der JNR kaum und ihr Anteil fiel auf $14 \%$. Die von der Bahn nicht mehr zu erbringende Leistung wurde im Personenverkehr von Bussen und PKW (1974: 17, resp. 33\% Anteil) und im Güterverkehr von LKWs und der Küstenschiffahrt (1974: 35, resp. 51\% Anteil) übernommen. Damit zeigt die japanische Verkehrsstruktur hinsichtlich der Aufteilung der Verkehrsaufgaben das ungewöhnliche Bild, dass den Bahnen der Passagierverkehr zu einem guten Teil verblieb, der Güterverkehr, der üblicherweise der finanziell tragende Teil ist, dagegen verloren ging. Der Bedeutungsschwund der Bahnen war in erster Linie dadurch bedingt, dass ihre Kapazität im Verhältnis zu den zu bewältigenden Aufgaben nicht oder ungenügend erweitert worden war. Lediglich im Personenverkehr erfolgten bei Shin-kan-sen entsprechende Investierungen und Erweiterungen. Beim Güterverkehr wurde erst in den Siebzigerjahren versucht, durch einen automatisierten Containerverkehr Bahnkunden zu gewinnen. Eine gewisse Leistungssteigerung der Bahnen wurde auch dadurch erzielt, dass die Dampftraktion durch Diesel und Elektrizität ersetzt wurde. Dies brachte freilich eine Verschlechterung der Zahlungsbilanz und eine erhöhte Auslandsabhängigkeit mit sich (siehe Beitrag in GH 2/77). 1975 wurden die letzten Dampflokomotiven ( $\mathrm{SL}=$ steam locomotives) aus dem Verkehr gezogen. Das Japanische kennt den Ausdruck "SL - mania" und wenn irgendwo einmal ein SL verkehrt, kommen Hunderte und Tausende von begeisterten SL-Fans zusammen, um das Ereignis in Bild und Ton festzuhalten.

Die stark gesteigerte Küstenschiffahrt führte an bestimmten Orten im japanischen Mittelmeer (Seto-nai-kai) schon dazu, dass auch hier die Kapazitätsgrenze erreicht wurde. Noch ausgesprochener ist dies beim Strassenverkehr der Fall. Obwohl seit 1955 ein ganzes System von Autobahnen gebaut und die wichtigsten Hauptstrassen verbessert wurden, setzen Stauungen, Langsamfahrt und Unfälle die tatsächliche Transportleistung im Verhältnis zur $\mathrm{Ka}-$ pazität immer mehr herunter.

Es besteht kein Zweifel darüber, dass mit Ausnahme einiger Lokallinien der JNR alle Teile des Verkehrssystemes - und nicht nur die Bahnen - völlig überlastet sind. Weil aber ein weiteres Wachstum der Wirtschaft angestrebt und erwartet wird, müssen die vorhandenen Strukturen verbessert und ergänzt werden. Sollte - was zu erwarten ist - die Stras se in Zukunft grössere Anteile an den Trans portleistungen übernehmen müssen, werden sich zusätzlich zu den angedeuteten Problemen weitere ergeben. Mann kann einen LKW als einen "automobilen Container", der ein bis zwei Mann Bedienung benötigt, betrachten. Die Voraussagen über die Entwicklung der Arbeitsjahrgänge lassen den Schluss zu, dass bald jener Punkt erreicht sein dürfte, an dem der Mangel an Fahrpersonal für LKWs als entscheidender Grenzfaktor wirksam werden wird. Man sollte auch beachten, dass bei der Strasse im Vergleich mit Bahn und Schiff der Leistungsabfall bei Annäherung an die maximale Kapazität schon früher und viel rascher einsetzt.

An Plänen für eine Verbesserung der Verkehrsstruktur fehlt es freilich nicht, und Vorarbeiten dazu sind auf technischem wie gesetzgeberischem Gebiet weit gediehen. Die Realisierung dieser Pläne wird -soweit es die JNR betrifft - dadurch erschwert, dass die JNR heute abgrundtief verschuldet sind. Schuld daran trägt weniger das Management als vielmehr der früher erwähnte Umstand, dass Tarifanpassungen durch das Parlament vorgenommen werden müssen. Dieses befass te sich aber mit der Lockheed-Affäre und anderen Skandalen und liess Sachgeschäfte wie JNR-Tarife liegen. Heute ist ein Zustand erreicht, wo selbst zweimalige Tariferhöhungen von je $50 \%$ (!) nur gerade aus dem Schlimmsten heraushelfen.

Trotz der üblen Finanzlage wurde eine Ausdehnung des Shin-kan-sen Systemes über sämtliche Inseln (total $7000 \mathrm{~km}$ ) geplant und streckenweise die Ausführung an die Hand genommen. 1976 wurde an folgenden Linien gebaut (Fig. 2): Die Tohoku-Linie welche vorerst bis Morioka, später bis Aomori und Sapporo geführt werden soll; die Joetsu-Linie von Tokyo nach Niigata an der Japanischen See; die kurze Strecke von Tokyo zum neuen internationalen Flugplatz Narita. Ein wichtiges 
Teilstück der Linie nach Sapporo ist der untermeerische Sei-kan Tunnel von Honshu unter der Tsugaru Strasse nach Hokkaido mit einer Länge von $53.85 \mathrm{~km}$, davon $23.3 \mathrm{~km}$ unter dem Meere. Die grössten Schwierigkeiten dürften bei den genannten Ausbauplänen nicht jene technischer Natur sein. Weil die Züge der Shin-kan-sen bei hohen Geschwindigkeiten starken Lärm verursachen, können die technisch möglichen Geschwindigkeiten vielerorts trotz Schallwänden mit Rücksicht auf die Bevölkerung nicht ausgenutzt werden. Alle drei genannten und im Bau begriffenen Linien müssen in die Grossstadt Tokyo hinein geführt und mit der nach Süden gehenden Shin-kan-sen verbunden werden. Solchen Vorhaben widersetzt sich die lokale Bevölkerung zusammen mit den lokalen Behörden. 1977 wird der internationale Flughafen Narita eröfnet und noch ist die Verbindungsbahn zur Stadt nicht gebaut. Auch der Sei-kan Tunnel scheint einige Jahre früher fertig zu sein als die Bahnen, denen er zu dienen hat. Der ausländische Beobachter nimmt solche Entwicklungen etwas verwirrt zur Kenntnis. Er sollte beachten, dass Entscheidungsprozesse in Japan anders als im Westen ablaufen. Schwierigkeiten werden Schritt um Schritt aus dem Wege geräumt und selbst finanzielle Probleme werden durchaus pragmatisch angegangen.

Dies zeigt sich auch deutlich bei den projektierten Brückenverbindungen zwischen Honshu und Shikoku. Heute werden diese Verbindungen durch Fähren (und Flugverkehr) hergestellt. Shikoku mit rund 4 Millionen Einwohnern steht trotz zahlreicher moderner Industrien an der Nordküste in der wirtschaftlichen Entwicklung hintennach. Die Brückenverbindungen sollen aber nicht nur Shikoku der wirtschaftlichen Zentralregion näherbringen, sie müssen auch als Alternative zu den bestehenden Verbindungen nach Kyushu gesehen werden. Aus der Vielzahl von Projekten gingen drei in die engere Wahl. Sie sind auf Fig. 3 mit den Buchstaben A, D und E bezeichnet. Rute A wird neben der Strasse zwei Geleise Schmalspur der JNR aufnehmen und besitzt mit der Akashi Kaikyo Brücke $(3560 \mathrm{~km})$ den längsten Brükkenschlag. Rute D wird ausser der Strasse zwei Geleise Schmal- und zwei Normalspur (Shin-kan-sen) aufweisen, während Rute E lediglich dem Strassenverkehr dienen wird. Die technischen Probleme werden heute als gelöst angesehen. Ernst werden dagegen die Einwände des Umweltschutzes - grosse Teile der Inlandsee sind Nationalparks - und die Frage der Entschädigungen an die durch den Brückenbau betroffenen Schiffahrtsinteressen genommen. Schrittweise geht man zur Zeit daran, die auftretenden Schwierigkeiten aus dem Wege zu räumen; 1976 sprach man dagegen von den finanziellen Problemen vorerst noch nicht. Dagegen beschloss die Regierung, dass vorerst nur die Rute D von Okayama. nach Sakaide an die Hand zu nehmen sei. Dieser Entscheid ist dafür verantwortlich, dass an dieser Rute noch nicht gebaut werden darf, denn noch sind nicht alle Probleme geklärt. Dagegen wurde an den beiden andern Ruten $\mathrm{A}$ und $\mathrm{E}$ die Arbeit an den beiden Brücken von Ōnaruto und Ömishima vorwärtsgetrieben. Bei allen drei Ruten war der Baubeginn ursprünglich auf 1974 angesetzt gewesen, doch konnte er nicht eingehalten werden.

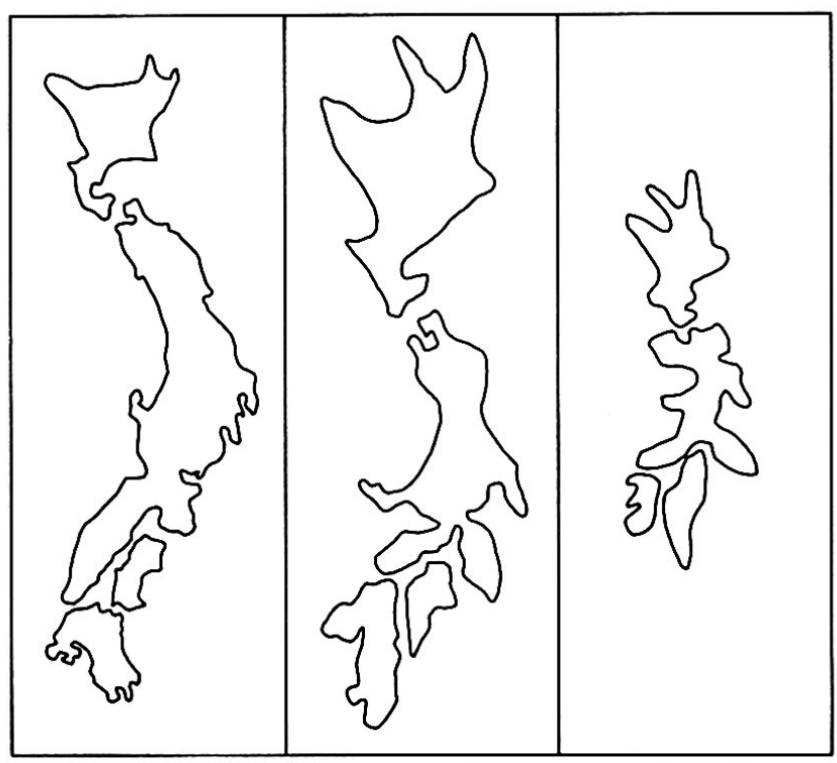

Fig. 1: Die durch Shin-kan-sen stark verkürzten Fahrzeiten bringen die großen Städte einander näher und verändern so die Dimensionen der japanischen Inseln in der Vorstellung der Japaner. Links: Die Dimensionen gegeben durch Länge und Breite, Mitte: Situation 1975, Rechts: Nach Ausbau der geplanten 7000 km Shin-kan-sen. Quelle: JNR, März 1975. 


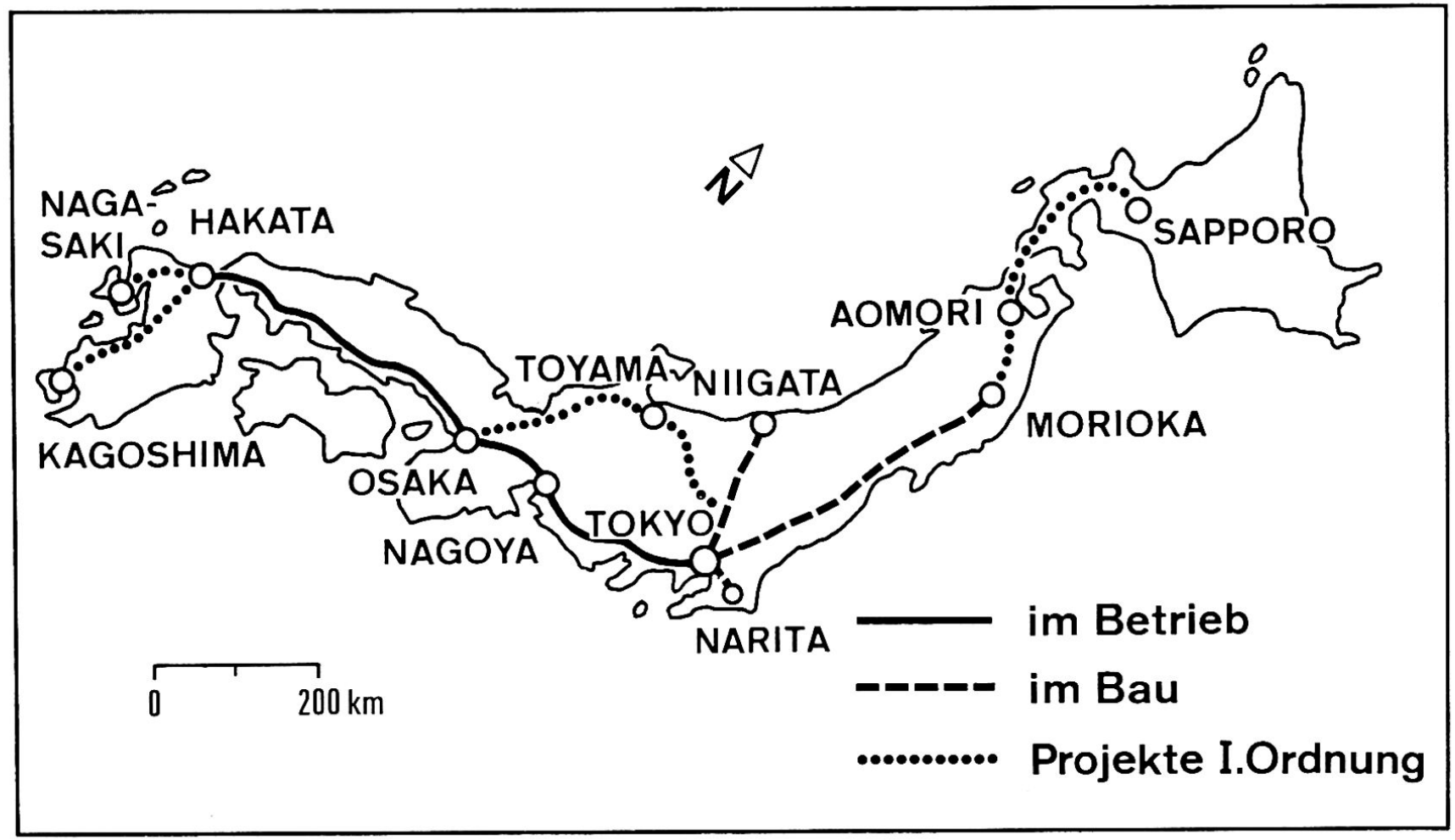

Fig. 2: Stand des Ausbaues des Shin-kan-sen Eisenbahnnetzes im März 1975, nach JNR.

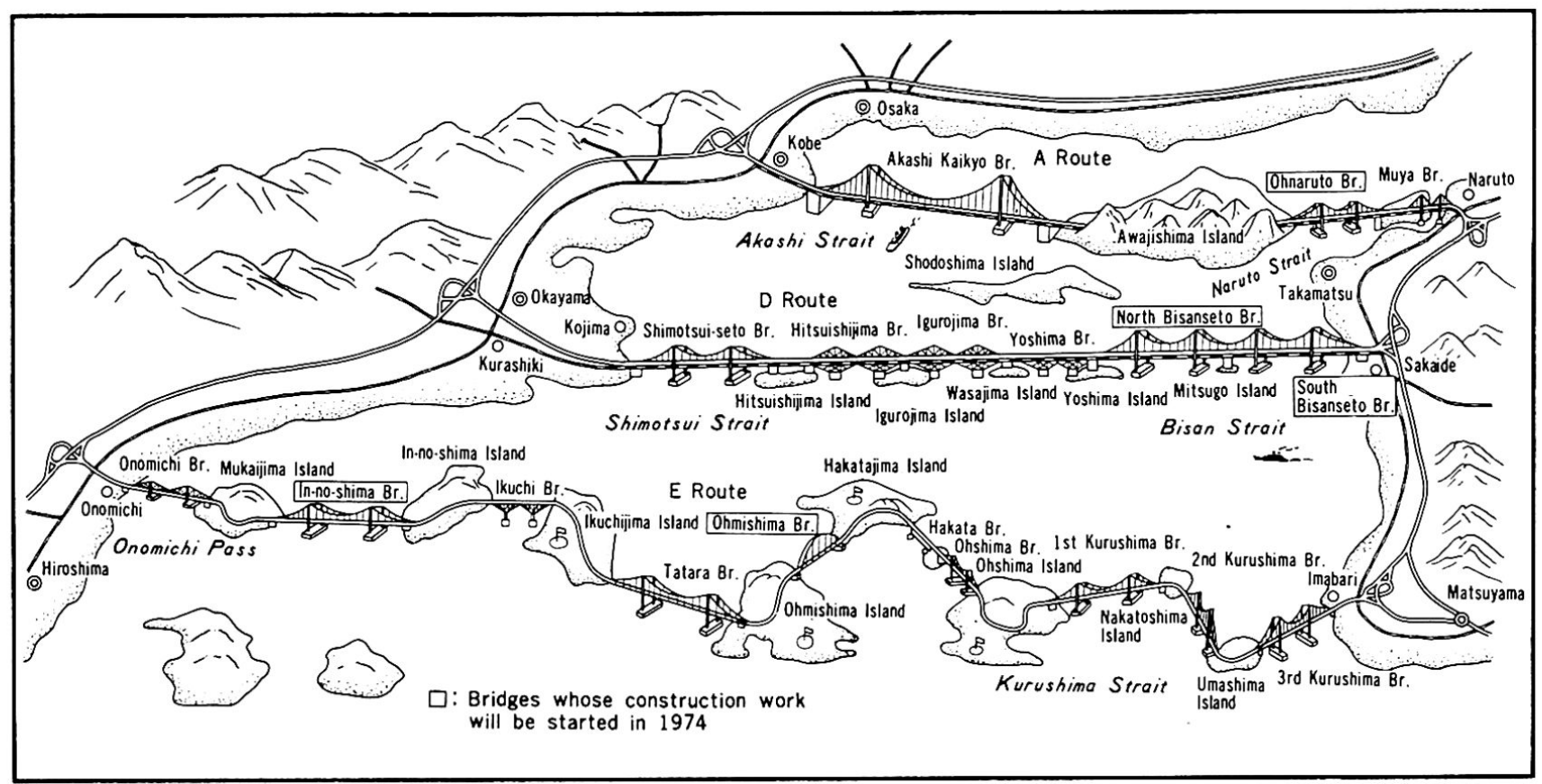

Fig. 3: Die drei Brückenprojekte von Honshu (links) nach Shikoku (rechts). Quelle: Honshu-Shikoku Bridge Authority, 1974. 